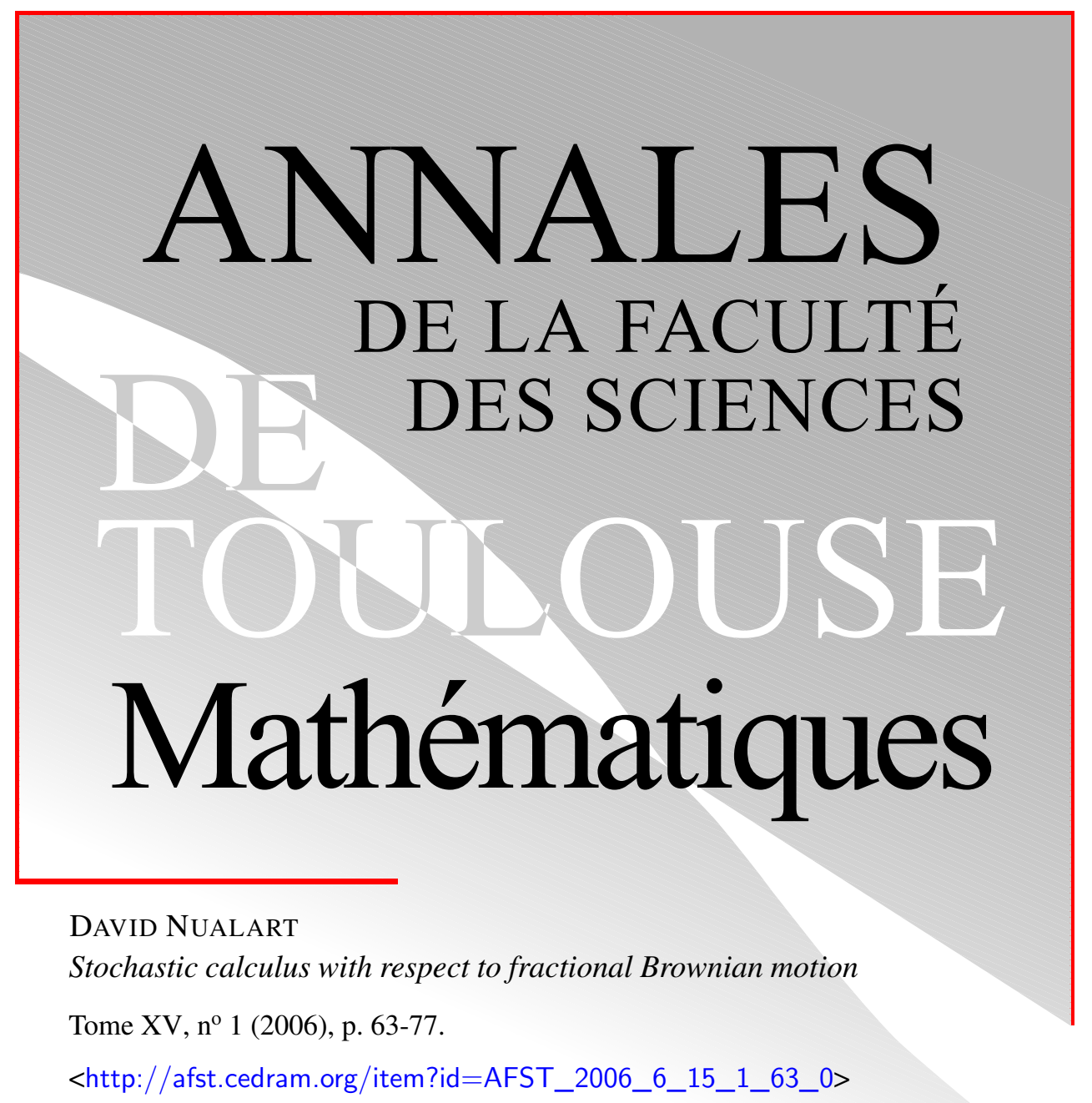

(C) Annales de la faculté des sciences de Toulouse Mathématiques, 2006, tous droits réservés.

L'accès aux articles de la revue "Annales de la faculté des sciences de Toulouse, Mathématiques » (http://afst.cedram.org/), implique l'accord avec les conditions générales d'utilisation (http://afst.cedram. org/legal/). Toute reproduction en tout ou partie cet article sous quelque forme que ce soit pour tout usage autre que l'utilisation à fin strictement personnelle du copiste est constitutive d'une infraction pénale. Toute copie ou impression de ce fichier doit contenir la présente mention de copyright.

\title{
cedram
}

Article mis en ligne dans le cadre du

Centre de diffusion des revues académiques de mathématiques

http://www.cedram.org/ 


\title{
Stochastic calculus with respect to fractional Brownian motion $\left.{ }^{*}\right)$
}

\author{
DAVID Nualart ${ }^{(1)}$
}

\begin{abstract}
Fractional Brownian motion (fBm) is a centered selfsimilar Gaussian process with stationary increments, which depends on a parameter $H \in(0,1)$ called the Hurst index. In this conference we will survey some recent advances in the stochastic calculus with respect to $\mathrm{fBm}$. In the particular case $H=1 / 2$, the process is an ordinary Brownian motion, but otherwise it is not a semimartingale and Itô calculus cannot be used. Different approaches have been introduced to construct stochastic integrals with respect to fBm: pathwise techniques, Malliavin calculus, approximation by Riemann sums. We will describe these methods and present the corresponding change of variable formulas. Some applications will be discussed.
\end{abstract}

RÉSUMÉ. - Le mouvement brownien fractionnaire (MBF) est un processus gaussien centré auto-similaire à accroissements stationnaires qui dépend d'un paramètre $H \in(0,1)$, appelé paramètre de Hurst. Dans cette conférence, nous donnerons une synthèse des résultats nouveaux en calcul stochastique par rapport à un MBF. Dans le cas particulier $H=1 / 2$, ce processus est le mouvement brownien classique, sinon, ce n'est pas une semi-martingale et on ne peut pas utiliser le calcul d'Itô. Différentes approches ont été utilisées pour construire des intégrales stochastiques par rapport à un MBF : techniques trajectorielles, calcul de Malliavin, approximation par des sommes de Riemann. Nous décrivons ces méthodes et présentons les formules de changement de variables associées. Plusieurs applications seront présentées.

\section{Fractional Brownian motion}

Fractional Brownian motion is a centered Gaussian process $B=\left\{B_{t}, t \geqslant 0\right\}$ with the covariance function

$$
R_{H}(t, s)=E\left(B_{t} B_{s}\right)=\frac{1}{2}\left(s^{2 H}+t^{2 H}-|t-s|^{2 H}\right) .
$$

(*) Reçu le 22 octobre 2004, accepté le 23 février 2005

(1) Facultat de Matemàtiques, Universitat de Barcelona, Gran Via 585, 08007 Barcelona (Spain). E-mail: dnualart@ub.edu 
The parameter $H \in(0,1)$ is called the Hurst parameter. This process was introduced by Kolmogorov [21] and studied by Mandelbrot and Van Ness in [24], where a stochastic integral representation in terms of a standard Brownian motion was established.

Fractional Brownian motion has the following self-similar property: For any constant $a>0$, the processes $\left\{a^{-H} B_{a t}, t \geqslant 0\right\}$ and $\left\{B_{t}, t \geqslant 0\right\}$ have the same distribution.

From (1.1) we can deduce the following expression for the variance of the increment of the process in an interval $[s, t]$ :

$$
E\left(\left|B_{t}-B_{s}\right|^{2}\right)=|t-s|^{2 H} .
$$

This implies that fBm has stationary increments. Furthermore, by Kolmogorov's continuity criterion, we deduce that $\mathrm{fBm}$ has a version with $\alpha$ Hölder continuous trajectories, for any $\alpha<H$.

For $H=\frac{1}{2}$, the covariance can be written as $R_{\frac{1}{2}}(t, s)=t \wedge s$, and the process $B$ is an ordinary Brownian motion. In this case the increments of the process in disjoint intervals are independent. However, for $H \neq \frac{1}{2}$, the increments are not independent, and, furthermore, the $\mathrm{fBm}$ is not a semimartingale. Let $r(n):=E\left[\left(B_{t+1}-B_{t}\right)\left(B_{n+1}-B_{n}\right)\right]$. Then, $r(n)$ behaves as $C n^{2 H-2}$, as $n$ tends to infinity (long-memory process). In particular, if $H>\frac{1}{2}$, then $\sum_{n}|r(n)|=\infty$ (long-range dependence) and if $H<\frac{1}{2}$, then, $\sum_{n}|r(n)|<\infty$ (short-range dependence).

The self-similarity and long memory properties make the fractional Brownian motion a suitable input noise in a variety of models. Recently, fBm has been applied in connection with financial time series, hydrology and telecommunications. In order to develop these applications there is a need for a stochastic calculus with respect to the fBm. Nevertheless, $\mathrm{fBm}$ is neither a semimartingale nor a Markov process, and new tools are required in order to handle the differentials of fBm and to formulate and solve stochastic differential equations driven by a $\mathrm{fBm}$.

There are essentially two different methods to define stochastic integrals with respect to the fractional Brownian motion:

(i) A path-wise approach that uses the Hölder continuity properties of the sample paths, developed from the works by Ciesielski, Kerkyacharian and Roynette [7] and Zähle [37].

(ii) The stochastic calculus of variations (Malliavin calculus) for the fBm introduced by Decreusefond and Üstünel in [13]. 
The stochastic calculus with respect to the fBm permits to formulate and solve stochastic differential equations driven by a $\mathrm{fBm}$. The stochastic integral defined using the Malliavin calculus leads to anticipative stochastic differential equations, which are difficult to solve except in some simple cases. In the one-dimensional case, the existence and uniqueness of a solution can be recovered by using the change-of-variable formula and the DossSussmanm method (see [26]). In the multidimensional case, when $H>\frac{1}{2}$, the existence and uniqueness of a solution have been established in several papers (see Lyons [22] and Nualart and Rascanu [28]). For $H \in\left(\frac{1}{4}, \frac{1}{2}\right)$, Coutin and Qian have obtained in [12] the existence of strong solutions and a Wong-Zakai type approximation limit for multi-dimensional stochastic differential equations driven by a $\mathrm{fBm}$, using the approach of rough path analysis developed by Lyons and Qian in [23]. The large deviations for these equations have been studied by Millet and Sanz-Solé in [25].

The purpose of this talk is to introduce some of the recent advances in the stochastic calculus with respect to the $\mathrm{fBm}$ and discuss several applications.

\section{Stochastic integration with respect to fractional Brownian motion}

We first construct the stochastic integral of deterministic functions.

\subsection{Wiener integral with respect to $\mathrm{fBm}$}

Fix a time interval $[0, T]$. Consider a $\mathrm{fBm}\left\{B_{t}, t \in[0, T]\right\}$ with Hurst parameter $H \in(0,1)$. We denote by $\mathcal{E}$ the set of step functions on $[0, T]$. Let $\mathcal{H}$ be the Hilbert space defined as the closure of $\mathcal{E}$ with respect to the scalar product

$$
\left\langle\mathbf{1}_{[0, t]}, \mathbf{1}_{[0, s]}\right\rangle_{\mathcal{H}}=R_{H}(t, s) .
$$

The mapping $\mathbf{1}_{[0, t]} \longrightarrow B_{t}$ can be extended to an isometry between $\mathcal{H}$ and the Gaussian space $H_{1}(B)$ associated with $B$. We will denote this isometry by $\varphi \longrightarrow B(\varphi)$, and we would like to interpret $B(\varphi)$ as the Wiener integral of $\varphi \in \mathcal{H}$ with respect to $B$ and to write $B(\varphi)=\int_{0}^{T} \varphi d B$. However, we do not know whether the elements of $\mathcal{H}$ can be considered as real-valued functions. This turns out to be true for $H<\frac{1}{2}$, but is false when $H>\frac{1}{2}$ (see Pipiras and Taqqu [30], [31]).

The $\mathrm{fBm}$ has the following integral representation:

$$
B_{t}=\int_{0}^{t} K_{H}(t, s) d W_{s}
$$




\section{David Nualart}

where $W=\left\{W_{t}, t \geqslant 0\right\}$ is an ordinary Wiener process and $K_{H}(t, s)$ is the Volterra kernel given by

$K_{H}(t, s)=c_{H}\left[\left(\frac{t}{s}\right)^{H-\frac{1}{2}}(t-s)^{H-\frac{1}{2}}-\left(H-\frac{1}{2}\right) s^{\frac{1}{2}-H} \int_{s}^{t} u^{H-\frac{3}{2}}(u-s)^{H-\frac{1}{2}} d u\right]$,

if $s<t$ and $K_{H}(t, s)=0$ if $s \geqslant t$. Here $c_{H}$ is the normalizing constant

$$
c_{H}=\left[\frac{\left(2 H+\frac{1}{2}\right) \Gamma\left(\frac{1}{2}-H\right)}{\Gamma\left(\frac{1}{2}+H\right) \Gamma(2-2 H)}\right]^{1 / 2} .
$$

The operator $K_{H}^{*}: \mathcal{E} \rightarrow L^{2}([0, T])$ defined by

$$
\left(K_{H}^{*} \mathbf{1}_{[0, t]}\right)(s)=K_{H}(t, s) .
$$

is a linear isometry that can be extended to the Hilbert space $\mathcal{H}$. In fact, for any $s, t \in[0, T]$ we have, using (2.3) and (2.1),

$$
\begin{aligned}
\left\langle K_{H}^{*} \mathbf{1}_{[0, t]}, K_{H}^{*} \mathbf{1}_{[0, s]}\right\rangle_{L^{2}([0, T])} & =\left\langle K_{H}(t, \cdot), K_{H}(s, \cdot)\right\rangle_{L^{2}([0, T])} \\
& =\int_{0}^{t \wedge s} K_{H}(t, u) K_{H}(s, u) d u \\
& =R_{H}(t, s)=\left\langle\mathbf{1}_{[0, t]}, \mathbf{1}_{[0, s]}\right\rangle_{\mathcal{H}} .
\end{aligned}
$$

This operator plays a basic role in the construction of a stochastic calculus with respect to $B$.

If $H>\frac{1}{2}$, the operator $K_{H}^{*}$ can be expressed in terms of fractional integrals:

$$
\left(K_{H}^{*} \varphi\right)(s)=c_{H} \Gamma\left(H-\frac{1}{2}\right) s^{\frac{1}{2}-H}\left(I_{T-}^{H-\frac{1}{2}} u^{H-\frac{1}{2}} \varphi(u)\right)(s),
$$

and $\mathcal{H}$ is the space of distributions $f$ such that $s^{\frac{1}{2}-H} I_{0+}^{H-\frac{1}{2}}\left(f(u) u^{H-\frac{1}{2}}\right)(s)$ is a square integrable function. In this case, the scalar product in $\mathcal{H}$ has the simpler expression

$$
\langle\varphi, \psi\rangle_{\mathcal{H}}=\alpha_{H} \int_{0}^{T} \int_{0}^{T}|r-u|^{2 H-2} \varphi_{r} \psi_{u} d u d r
$$

where $\alpha_{H}=H(2 H-1)$, and $\mathcal{H}$ contains the Banach space $|\mathcal{H}|$ of measurable functions $\varphi$ on $[0, T]$ such that

$$
\|\varphi\|_{|\mathcal{H}|}^{2}=\alpha_{H} \int_{0}^{T} \int_{0}^{T}\left|\varphi_{r}\right|\left|\varphi_{u}\right||r-u|^{2 H-2} d r d u<\infty .
$$


We have the following continuous embeddings (see [31]):

$$
L^{\frac{1}{H}}([0, T]) \subset|\mathcal{H}| \subset \mathcal{H} .
$$

For $H<\frac{1}{2}$, the operator $K_{H}^{*}$ can be expressed in terms of fractional derivatives:

$$
\left(K_{H}^{*} \varphi\right)(s)=d_{H} s^{\frac{1}{2}-H}\left(D_{T-}^{\frac{1}{2}-H} u^{H-\frac{1}{2}} \varphi(u)\right)(s),
$$

where $d_{H}=c_{H} \Gamma\left(H+\frac{1}{2}\right)$. In this case, $\mathcal{H}=I_{T-}^{\frac{1}{2}-H}\left(L^{2}\right)$ (see [13]) and

$$
C^{\gamma}([0, T]) \subset \mathcal{H}
$$

if $\gamma>\frac{1}{2}-H$.

As a consequence, we deduce the following transfer rule:

$$
B(\varphi)=W\left(K_{H}^{*} \varphi\right),
$$

for any $\varphi \in \mathcal{H}$.

\subsection{Stochastic integrals of random processes}

Suppose now that $u=\left\{u_{t}, t \in[0, T]\right\}$ is a random process. By the transfer rule (2.7) we can write

$$
\int_{0}^{T} u_{t} d B_{t}=\int_{0}^{T}\left(K_{H}^{*} u\right)_{t} d W_{t}
$$

However, even if the process $u$ is adapted to the filtration generated by the $\mathrm{fBm}$ (which coincides with the filtration generated by $W$ ), the process $K_{H}^{*} u$ is no longer adapted because the operator $K_{H}^{*}$ does not preserves the adaptability. Therefore, in order to define stochastic integrals of random processes with respect to the $\mathrm{fBm}$ we need anticipating integrals.

In the case of an ordinary Brownian motion, the divergence operator coincides with an extension of Itô's stochastic integral to anticipating processes introduced by Skorohod in [34]. Thus, we could use the Skorohod integral in formula (2.8), and in that case, the integral $\int_{0}^{T} u_{t} d B_{t}$ coincides with the divergence operator in the Malliavin calculus with respect to the $\mathrm{fBm} B$. The approach of Malliavin calculus to define stochastic integrals with respect to the fBm has been introduced by Decreusefont and Üstünel in [13], and further developed by several authors (Carmona and Coutin [6], Alòs, Mazet and Nualart [3], Alòs and Nualart [4], Alòs, León and Nualart [1], and $\mathrm{Hu}[18])$. 


\section{David Nualart}

\subsubsection{Stochastic calculus of variations with respect to $\mathrm{fBm}$}

Let $B=\left\{B_{t}, t \in[0, T]\right\}$ be a fBm with Hurst parameter $H \in(0,1)$. Let $\mathcal{S}$ be the set of smooth and cylindrical random variables of the form

$$
F=f\left(B\left(\phi_{1}\right), \ldots, B\left(\phi_{n}\right)\right),
$$

where $n \geqslant 1, f \in \mathrm{C}_{b}^{\infty}\left(\mathbb{R}^{n}\right)$ ( $f$ and all its partial derivatives are bounded), and $\phi_{i} \in \mathcal{H}$.

The derivative operator $D$ of a smooth and cylindrical random variable $F$ of the form (2.9) is defined as the $\mathcal{H}$-valued random variable

$$
D F=\sum_{i=1}^{n} \frac{\partial f}{\partial x_{i}}\left(B\left(\phi_{1}\right), \ldots, B\left(\phi_{n}\right)\right) \phi_{i} .
$$

The derivative operator $D$ is then a closable operator from $L^{2}(\Omega)$ into $L^{2}(\Omega ; \mathcal{H})$. We denote by $\mathbb{D}^{1,2}$ is the closure of $\mathcal{S}$ with respect to the norm

$$
\|F\|_{1,2}=\sqrt{E\left(F^{2}\right)+E\left(\|D F\|_{\mathcal{H}}^{2}\right)} .
$$

The divergence operator $\delta$ is the adjoint of the derivative operator. That is, we say that a random variable $u$ in $L^{2}(\Omega ; \mathcal{H})$ belongs to the domain of the divergence operator, denoted by Dom $\delta$, if

$$
\left|E\left(\langle D F, u\rangle_{\mathcal{H}}\right)\right| \leqslant c_{u}\|F\|_{L^{2}(\Omega)}
$$

for any $F \in \mathcal{S}$. In this case $\delta(u)$ is defined by the duality relationship

$$
E(F \delta(u))=E\left(\langle D F, u\rangle_{\mathcal{H}}\right),
$$

for any $F \in \mathbb{D}^{1,2}$.

We have $\mathbb{D}^{1,2}(\mathcal{H}) \subset$ Dom $\delta$ and for any $u \in \mathbb{D}^{1,2}(\mathcal{H})$

$$
E\left(\delta(u)^{2}\right)=E\left(\|u\|_{\mathcal{H}}^{2}\right)+E\left(\left\langle D u,(D u)^{*}\right\rangle_{\mathcal{H} \otimes \mathcal{H}}\right),
$$

where $(D u)^{*}$ is the adjoint of $(D u)$ in the Hilbert space $\mathcal{H} \otimes \mathcal{H}$.

\subsubsection{The divergence and symmetric integrals in the case $H>\frac{1}{2}$}

The following result (see [4]) provides a relationship between the divergence operator and the symmetric stochastic integral introduced by Russo and Vallois in [33]. 
Stochastic calculus with respect to the fractional Brownian motion and applications

Proposition 2.1. - Let $u=\left\{u_{t}, t \in[0, T]\right\}$ be a stochastic process in the space $\mathbb{D}^{1,2}(\mathcal{H})$. Suppose that

$$
E\left(\|u\|_{|\mathcal{H}|}^{2}+\|D u\|_{|\mathcal{H}| \otimes|\mathcal{H}|}^{2}\right)<\infty
$$

and

$$
\int_{0}^{T} \int_{0}^{T}\left|D_{s} u_{t}\right||t-s|^{2 H-2} d s d t<\infty, a . s .
$$

Then the symmetric integral $\int_{0}^{T} u_{t} d B_{t}$, defined as the limit in probability as $\varepsilon$ tends to zero of

$$
(2 \varepsilon)^{-1} \int_{0}^{T} u_{s}\left(B_{(s+\varepsilon) \wedge T}-B_{(s-\varepsilon) \vee 0}\right) d s,
$$

exists and we have

$$
\int_{0}^{T} u_{t} d B_{t}=\delta(u)+\alpha_{H} \int_{0}^{T} \int_{0}^{T} D_{s} u_{t}|t-s|^{2 H-2} d s d t .
$$

Remark. - The symmetric integral can be replaced by the forward or backward integrals in the above proposition.

Suppose that $u=\left\{u_{t}, t \in[0, T]\right\}$ is a stochastic process satisfying the conditions of Proposition 2.1. Then, we can define the indefinite integral $\int_{0}^{t} u_{s} d B_{s}=\int_{0}^{T} u_{s} \mathbf{1}_{[0, t]}(s) d B_{s}$ and the following decomposition holds

$$
\int_{0}^{t} u_{s} d B_{s}=\delta\left(u \mathbf{1}_{[0, t]}\right)+\alpha_{H} \int_{0}^{t} \int_{0}^{T} D_{r} u_{s}|s-r|^{2 H-2} d r d s .
$$

The second summand in this expression is a process with absolutely continuous paths. The first summand can be estimated using Meyer's inequalities for the divergence operator. For any $p>1$, we denote by $\mathbb{L}_{H}^{1, p}$ is the set of processes $u \in \mathbb{D}^{1, p}(\mathcal{H})$ such that

$$
\|u\|_{\mathbb{L}_{H}^{1, p}}^{p}:=E\left(\|u\|_{L^{1 / H}([0, T])}^{p}+\|D u\|_{L^{1 / H}\left([0, T]^{2}\right)}^{p}\right)<\infty .
$$

If $u \in \mathbb{L}_{H}^{1, p}$ with $p H>1$ and

$$
\|u\|_{1, p}^{p}:=\int_{0}^{T}\left|E\left(u_{s}\right)\right|^{p} d s+\int_{0}^{T} E\left(\int_{0}^{T}\left|D_{s} u_{r}\right|^{1 / H} d s\right)^{p H} d r<\infty
$$


then the indefinite divergence integral $X_{t}=\int_{0}^{t} u_{s} \delta B_{s}$ has a version with $\gamma$-Hölder continuous trajectories and for all $\gamma<H-\frac{1}{p}$ and the following maximal inequality holds

$$
E\left(\sup _{t \in[0, T]}\left|\int_{0}^{t} u_{s} \delta B_{s}\right|^{p}\right) \leqslant C\|u\|_{1, p} .
$$

\subsubsection{Itô's formula for the divergence integral}

If $F$ is a function of class $C^{2}$, and $H>\frac{1}{2}$, the path-wise RiemannStieltjes integral $\int_{0}^{t} F^{\prime}\left(B_{s}\right) d B_{s}$ exists for each $t \in[0, T]$ by the theory of Young [36]. Moreover the following change of variables formula holds:

$$
F\left(B_{t}\right)=F(0)+\int_{0}^{t} F^{\prime}\left(B_{s}\right) d B_{s} .
$$

Suppose that $F$ is a function of class $C^{2}(\mathbb{R})$ such that

$$
\max \left\{|F(x)|,\left|F^{\prime}(x)\right|,\left|F^{\prime \prime}(x)\right|\right\} \leqslant c e^{\lambda x^{2}},
$$

where $c$ and $\lambda$ are positive constants such that $\lambda<\frac{1}{4 T^{2 H}}$. Then, the process $F^{\prime}\left(B_{t}\right)$ satisfies the conditions of Proposition 2.1. As a consequence, we obtain

$$
\begin{aligned}
\int_{0}^{t} F^{\prime}\left(B_{s}\right) d B_{s} & =\int_{0}^{t} F^{\prime}\left(B_{s}\right) \delta B_{s}+\alpha_{H} \int_{0}^{t} \int_{0}^{s} F^{\prime \prime}\left(B_{s}\right)(s-r)^{2 H-2} d r d s \\
& =\int_{0}^{t} F^{\prime}\left(B_{s}\right) \delta B_{s}+H \int_{0}^{t} F^{\prime \prime}\left(B_{s}\right) s^{2 H-1} d s .
\end{aligned}
$$

Therefore, putting together (2.15) and (2.17) we deduce the following Itô's formula for the divergence process

$$
F\left(B_{t}\right)=F(0)+\int_{0}^{t} F^{\prime}\left(B_{s}\right) \delta B_{s}+H \int_{0}^{t} F^{\prime \prime}\left(B_{s}\right) s^{2 H-1} d s .
$$

The following general version of Itô's formula has been proved in [4]:

Theorem 2.2. - Let $F$ be a function of class $C^{2}(\mathbb{R})$. Assume that $u=\left\{u_{t}, t \in[0, T]\right\}$ is a process locally in the space $\mathbb{D}^{2,2}(|\mathcal{H}|)$ such that the indefinite integral $X_{t}=\int_{0}^{t} u_{s} \delta B_{s}$ is a.s. continuous. Assume that $\|u\|_{2}$ 
belongs to $\mathcal{H}$. Then for each $t \in[0, T]$ the following formula holds

$$
\begin{aligned}
F\left(X_{t}\right)= & F(0)+\int_{0}^{t} F^{\prime}\left(X_{s}\right) u_{s} \delta B_{s} \\
& +\alpha_{H} \int_{0}^{t} F^{\prime \prime}\left(X_{s}\right) u_{s}\left(\int_{0}^{T}|s-\sigma|^{2 H-2}\left(\int_{0}^{s} D_{\sigma} u_{\theta} \delta B_{\theta}\right) d \sigma\right) d s \\
& +\alpha_{H} \int_{0}^{t} F^{\prime \prime}\left(X_{s}\right) u_{s}\left(\int_{0}^{s} u_{\theta}(s-\theta)^{2 H-2} d \theta\right) d s .
\end{aligned}
$$

Remark. - Taking the limit as $H$ converges to $\frac{1}{2}$ in Equation (2.19) we recover the usual Itô's formula for the the Skorohod integral proved by Nualart and Pardoux [27].

The following result on the $p$-variation of the divergence integral has been obtained by in [17]. Fix $T>0$ and set $t_{i}^{n}:=\frac{i T}{n}$, where $n$ is a positive integer and $i=0,1, \ldots, n$. Given a stochastic process $X=\left\{X_{t}, t \in[0, T]\right\}$ and $p \geqslant 1$, we set

$$
V_{n}^{p}(X):=\sum_{i=0}^{n-1}\left|X_{t_{i+1}^{n}}-X_{t_{i}^{n}}\right|^{p}
$$

TheOREM 2.3. - Let $\frac{1}{2}<H<1$ and $u \in \mathbb{L}_{H}^{1,1 / H}$. Set $X_{t}:=\int_{0}^{t} u_{s} \delta B_{s}$, for each $t \in[0, T]$. Then

$$
V_{n}^{1 / H}(X) n \rightarrow \infty \underset{L^{1}(\Omega)}{\longrightarrow} C_{H} \int_{0}^{T}\left|u_{s}\right|^{1 / H} d s
$$

where $C_{H}:=E\left(\left|B_{1}\right|^{1 / H}\right)$.

\subsubsection{Stochastic integration with respect to $\mathrm{fBm}$ in the case $H<\frac{1}{2}$}

The extension of the previous results to the case $H<\frac{1}{2}$ is not trivial and new difficulties appear. For instance, the forward integral $\int_{0}^{T} B_{t} d B_{t}$ in the sense of Russo and Vallois does not exists, and one is forced to use symmetric integrals. A counterpart of Proposition 2.1 in the case $H<\frac{1}{2}$ and Itô's formulae 2.18 and 2.19 have been proved in [1] for $\frac{1}{4}<H<\frac{1}{2}$. The reason for the restriction $\frac{1}{4}<H$ is the following. In order to define the divergence integral $\int_{0}^{T} F^{\prime}\left(B_{s}\right) \delta B_{s}$, we need the process $F^{\prime}\left(B_{s}\right)$ to belong to $L^{2}(\Omega ; \mathcal{H})$. This is clearly true, provided $F$ satisfies the growth condition 


\section{David Nualart}

(2.16), because $F^{\prime}\left(B_{s}\right)$ is Hölder continuous of order $H-\varepsilon>\frac{1}{2}-H$ if $\varepsilon<2 H-\frac{1}{2}$. If $H \leqslant \frac{1}{4}$, one can show (see [9]) that

$$
P(B \in \mathcal{H})=0,
$$

and the space $\mathbb{D}^{1,2}(\mathcal{H})$ is too small to contain processes of the form $F^{\prime}\left(B_{t}\right)$.

In [9] a new approach is introduced in order to extend the domain of the divergence operator to processes whose trajectories are not necessarily in the space $\mathcal{H}$. The basic tool for this extension of the divergence operator is the adjoint of the operator $K_{H}^{*}$ in $L^{2}(0, T)$ given by

$$
\left(K_{H}^{*, a} \varphi\right)(s)=d_{H} s^{H-\frac{1}{2}} D_{0+}^{\frac{1}{2}-H}\left(u^{\frac{1}{2}-H} \varphi(u)\right)(s) .
$$

Set $\mathcal{H}_{2}=\left(K_{H}^{*}\right)^{-1}\left(K_{H}^{*, a}\right)^{-1}\left(L^{2}(0, T)\right)$ and denote by $\mathcal{S}_{\mathcal{H}}$ the space of smooth and cylindrical random variables of the form

$$
F=f\left(B\left(\phi_{1}\right), \ldots, B\left(\phi_{n}\right)\right),
$$

where $n \geqslant 1, f \in C_{b}^{\infty}\left(\mathbb{R}^{n}\right)$, and $\phi_{i} \in \mathcal{H}_{2}$.

Definition 2.4. - Let $u=\left\{u_{t}, t \in[0, T]\right\}$ be a measurable process such that

$$
E\left(\int_{0}^{T} u_{t}^{2} d t\right)<\infty .
$$

We say that $u \in \operatorname{Dom}^{*} \delta$ if there exists a random variable $\delta(u) \in L^{2}(\Omega)$ such that for all $F \in \mathcal{S}_{\mathcal{H}}$ we have

$$
\int_{\mathbb{R}} E\left(u_{t} K_{H}^{*, a} K_{H}^{*} D_{t} F\right) d t=E(\delta(u) F) .
$$

This extended domain of the divergence operator satisfies the following elementary properties:

1. $\operatorname{Dom} \delta \subset \operatorname{Dom}^{*} \delta$, and $\delta$ restricted to Dom $\delta$ coincides with the divergence operator.

2. If $u \in \operatorname{Dom}^{*} \delta$ then $E(u)$ belongs to $\mathcal{H}$.

3. If $u$ is a deterministic process, then $u \in \operatorname{Dom}^{*} \delta$ if and only if $u \in \mathcal{H}$. 
This extended domain of the divergence operator leads to the following version of Itô's formula for the divergence process, established by Cheridito and Nualart in [9].

THEOREM 2.5. - Suppose that $F$ is a function of class $C^{2}(\mathbb{R})$ satisfying the growth condition (2.16). Then for all $t \in[0, T]$, the process $\left\{F^{\prime}\left(B_{s}\right) \mathbf{1}_{[0, t]}(s)\right\}$ belongs to $\operatorname{Dom}^{*} \delta$ and we have

$$
F\left(B_{t}\right)=F(0)+\int_{0}^{t} F^{\prime}\left(B_{s}\right) \delta B_{s}+H \int_{0}^{t} F^{\prime \prime}\left(B_{s}\right) s^{2 H-1} d s .
$$

\subsubsection{Local time and Tanaka's formula for $\mathrm{fBm}$}

Berman proved in [5] that that fractional Brownian motion $B=\left\{B_{t}, t \geqslant 0\right\}$ has a local time $l_{t}^{a}$ continuous in $(a, t) \in \mathbb{R} \times[0, \infty)$ which satisfies the occupation formula

$$
\int_{0}^{t} g\left(B_{s}\right) d s=\int_{\mathbb{R}} g(a) l_{t}^{a} d a .
$$

for every continuous and bounded function $g$ on $\mathbb{R}$. Set

$$
L_{t}^{a}=2 H \int_{0}^{t} s^{2 H-1} l^{a}(d s) .
$$

Then $a \rightarrow L_{t}^{a}$ is the density of the occupation measure

$$
\mu(C)=2 H \int_{0}^{t} \mathbf{1}_{C}\left(B_{s}\right) s^{2 H-1} d s,
$$

where $C$ is a Borel subset of $\mathbb{R}$. As an extension of the Itô 's formula (2.22), the following result has been proved in [9]:

Theorem 2.6. - Let $0<t<\infty$ and $a \in \mathbb{R}$. Then

$$
\mathbf{1}_{\left\{B_{s}>a\right\}} \mathbf{1}_{[0, t]}(s) \in \operatorname{Dom}^{*} \delta,
$$

and

$$
\left(B_{t}-a\right)^{+}=(-a)^{+}+\int_{0}^{t} \mathbf{1}_{\left\{B_{s}>a\right\}} \delta B_{s}+\frac{1}{2} L_{t}^{a} .
$$

This result can be considered as a version of Tanaka's formula for the fBm. In [11] it is proved that for $H>\frac{1}{3}$, the process $\mathbf{1}_{\left\{B_{s}>a\right\}} \mathbf{1}_{[0, t]}(s)$ belongs to Dom $\delta$ and ( 2.24) holds. 


\section{Fractional Bessel processes}

Let $B=\left\{\left(B_{t}^{1}, \ldots, B_{t}^{d}\right), t \geqslant 0\right\}$ be a $d$-dimensional fractional Brownian motion with Hurst parameter $H \in(0,1)$. The fractional Bessel process is defined by $R_{t}=\sqrt{\left(B_{t}^{1}\right)^{2}+\cdots+\left(B_{t}^{d}\right)^{2}}$. If $H>\frac{1}{2}$ and $d \geqslant 2$, as an application of the multidimensional version of the Itô formula (2.18), one obtains (see [17]):

$$
R_{t}=\sum_{i=1}^{d} \int_{0}^{t} \frac{B_{s}^{i}}{R_{s}} \delta B_{s}^{i}+H(d-1) \int_{0}^{t} \frac{s^{2 H-1}}{R_{s}} d s .
$$

For $d=1$, Tanaka's formula (2.24) says that (for any $H \in(0,1)$ )

$$
\left|B_{t}\right|=\int_{0}^{t} \operatorname{sign}\left(B_{s}\right) \delta B_{s}+L_{t}^{0} .
$$

Assume $H>\frac{1}{2}$ and set

$$
X_{t}=\left\{\begin{array}{l}
\sum_{i=1}^{d} \int_{0}^{t} \frac{B_{s}^{i}}{R_{s}} \delta B_{s}^{i} \quad \text { if } \quad d \geqslant 2 \\
\int_{0}^{t} \operatorname{sign}\left(B_{s}\right) \delta B_{s} \quad \text { if } \quad d=1
\end{array} .\right.
$$

In the standard Brownian motion case, the process $X_{t}$ is a one-dimensional Brownian motion, as a consequence of Lévy's characterization theorem. The process $X_{t}$ is $H$ self-similar and it has the the same $\frac{1}{H}$-finite variation as the $\mathrm{fBm}$. It is then natural to conjecture that $X_{t}$ is a $\mathrm{fBm}$. Some partial results have been obtained so far:

It has been proved in [19] that $X_{t}$ is not an $\mathcal{F}_{t}$-fractional Brownian motion, where $\mathcal{F}_{t}$ is the filtration generated by the $\mathrm{fBm}$. Moreover, it is proved in [19] that for $H>2 / 3$ it does not have the long-range dependence property and, as a consequence, it is not a $\mathrm{fBm}$. In [14] it is proved that for any Hurst parameter $H \in(0,1), H \neq \frac{1}{2}$, it is not possible for the process $X_{t}$ defined in (3.3) to be a $\mathrm{fBm}$ and to safisfy the equation

$$
R_{t}^{2}=2 \int_{0}^{t} R_{s} \delta X_{s}+n t^{2 H} .
$$


Stochastic calculus with respect to the fractional Brownian motion and applications

\section{Vortex filaments based on fBm}

The observations of three-dimensional turbulent fluids indicate that the vorticity field of the fluid is concentrated along thin structures called vortex filaments. In his book Chorin [10] suggests probabilistic descriptions of vortex filaments by trajectories of self-avoiding walks on a lattice. Flandoli [15] introduced a model of vortex filaments based on a three-dimensional Brownian motion. A basic problem in these models is the computation of the kynetic energy of a given configuration.

Denote by $u(x)$ the velocity field of the fluid at point $x \in \mathbb{R}^{3}$, and let $\xi=$ curl $u$ be the associated vorticity field. The kynetic energy of the field will be

$$
\mathbb{H}=\frac{1}{2} \int_{\mathbb{R}^{3}}|u(x)|^{2} d x=\frac{1}{8 \pi} \int_{\mathbb{R}^{3}} \int_{\mathbb{R}^{3}} \frac{\xi(x) \cdot \xi(y)}{|x-y|} d x d y
$$

We will assume that the vorticity field is concentrated along a thin tube centered in a curve $\gamma=\left\{\gamma_{t}, 0 \leqslant t \leqslant T\right\}$. Moreover, we will choose a random model and consider this curve as the trajectory of a three-dimensional fractional Brownian motion $B=\left\{B_{t}, 0 \leqslant t \leqslant T\right\}$. This can be formally expressed as

$$
\xi(x)=\Gamma \int_{\mathbb{R}^{3}}\left(\int_{0}^{T} \delta\left(x-y-B_{s}\right) \cdot B_{s} d s\right) \rho(d y),
$$

where $\Gamma$ is a parameter called the circuitation, and $\rho$ is a probability measure on $\mathbb{R}^{3}$ with compact support.

Substituting (4.2) into (4.1) we derive the following formal expression for the kynetic energy:

$$
\mathbb{H}=\int_{\mathbb{R}^{3}} \int_{\mathbb{R}^{3}} \mathbb{H}_{x y} \rho(d x) \rho(d y),
$$

where the so-called interaction energy $\mathbb{H}_{x y}$ is given by the double integral

$$
\mathbb{H}_{x y}=\frac{\Gamma^{2}}{8 \pi} \sum_{i=1}^{3} \int_{0}^{T} \int_{0}^{T} \frac{1}{\left|x+B_{t}-y-B_{s}\right|} d B_{s}^{i} d B_{t}^{i}
$$

We are interested in the following problems: Is $\mathbb{H}$ a well defined random variable? Does it have moments of all orders and even exponential moments? 
In order to give a rigorous meaning to the double integral (4.4) let us introduce the regularization of the function $|\cdot|^{-1}$ :

$$
\sigma_{n}=|\cdot|^{-1} * p_{1 / n},
$$

where $p_{1 / n}$ is the Gaussian kernel with variance $\frac{1}{n}$. Then, the smoothed interaction energy

$$
\mathbb{H}_{x y}^{n}=\frac{\Gamma^{2}}{8 \pi} \sum_{i=1}^{3} \int_{0}^{T}\left(\int_{0}^{T} \sigma_{n}\left(x+B_{t}-y-B_{s}\right) d B_{s}^{i}\right) d B_{t}^{i},
$$

is well defined, where the integrals are path-wise Riemann-Stieltjes integrals. Set

$$
\mathbb{H}^{n}=\int_{\mathbb{R}^{3}} \int_{\mathbb{R}^{3}} \mathbb{H}_{x y}^{n} \rho(d x) \rho(d y) .
$$

The following result has been proved in [29]:

TheOREM 4.1. - Suppose that the measure $\rho$ satisfies

$$
\int_{\mathbb{R}^{3}} \int_{\mathbb{R}^{3}}|x-y|^{1-\frac{1}{H}} \rho(d x) \rho(d y)<\infty .
$$

Let $\mathbb{H}_{x y}^{n}$ be the smoothed interaction energy defined by (4.6). Then $\mathbb{H}^{n}$ defined in (4.7) converges, for all $k \geqslant 1$, in $L^{k}(\Omega)$ to a random variable $\mathbb{H} \geqslant 0$ that we call the energy associated with the vorticity field (4.2).

If $H=\frac{1}{2}$, $\mathrm{fBm} B$ is a classical three-dimensional Brownian motion. In this case condition (4.8) would be $\int_{\mathbb{R}^{3}} \int_{\mathbb{R}^{3}}|x-y|^{-1} \rho(d x) \rho(d y)<\infty$, which is the assumption made by Flandoli [15] and Flandoli and Gubinelli [16]. In this last paper, using Fourier approach and Itô's stochastic calculus, the authors show that $E e^{-\beta \mathbb{H}}<\infty$ for sufficiently small $\beta$.

The proof of Theorem 4.1 is based on the stochastic calculus of variations with respect to $\mathrm{fBm}$ and the application of Fourier transform. 
Stochastic calculus with respect to the fractional Brownian motion and applications

\section{Bibliography}

[1] Alòs (E.), León (J. A.), Nualart (D.). - Stratonovich stochastic calculus with respect to fractional Brownian motion with Hurst parameter less than 1/2. Taiwanesse Journal of Mathematics 5, p. 609-632 (2001).

[2] Alòs (E.), Mazet (O.), Nualart (D.). - Stochastic calculus with respect to fractional Brownian motion with Hurst parameter lesser than $\frac{1}{2}$. Stoch. Proc. Appl. 86, p. 121-139 (1999).

[3] Alòs (E.), Mazet (O.), Nualart (D.). - Stochastic calculus with respect to Gaussian processes. Annals of Probability 29, p. 766-801 (2001).

[4] Alòs (E.), Nualart (D.). - Stochastic integration with respect to the fractional Brownian motion. Stochastics and Stochastics Reports 75, p. 129-152 (2003).

[5] Berman (S.). - Local nondeterminism and local times of Gaussian processes. Indiana Univ. Math. J. 23, p. 69-94 (1973).

[6] Carmona (P.), Coutin (L.). - Stochastic integration with respect to fractional Brownian motion. Ann. Institut Henri Poincaré 39, p. 27-68 (2003).

[7] Ciesielski (Z.), Kerkyacharian (G.) and Roynette (B.). - Quelques espaces fonctionnels associés à des processus gaussiens. Studia Math. 107, p. 171-204 (1993).

[8] Cheridito (P.). — Mixed fractional Brownian motion. Bernoulli 7 (2001), p. 913934.

[9] Cheridito (P.), Nualart (D.). - Stochastic integral of divergence type with respect to the fractional Brownian motion with Hurst parameter $H<\frac{1}{2}$. Ann. Institut Henri Poincaré 41, p. 1049-1081 (2005).

[10] Chorin (A.). — Vorticity and Turbulence, Springer-Verlag, 1994.

[11] Coutin (L.), Nualart (D.), Tudor (C. A.). - Tanaka formula for the fractional Brownian motion. Stochastic Processes Appl. 94, p. 301-315 (2001).

[12] Coutin (L.) and QIAn (Z.). - Stochastic analysis, rough paths analysis and fractional Brownian motions. Probab. Theory Rel. Fields 122, p. 108-140 (2002).

[13] Decreusefond (L.), Üstünel (A. S.). - Stochastic analysis of the fractional Brownian motion. Potential Analysis 10, p. 177-214 (1998).

[14] Eisenbaum (N.), Tudor (C. A.). - On squared fractional Brownian motions. Lecture Notes in Math. 1857, p. 282-289 (2005).

[15] Flandoli (F.). - On a probabilistic description of small scale structures in 3D fluids. Ann. Inst. Henri Poincaré 38, p. 207-228 (2002).

[16] Flandoli (F.), Gubinelli (M.). - The Gibbs ensemble of a vortex filament. Probab. Theory Relat. Fields 122, p. 317-340 (2001).

[17] Guerra (J.), Nualart (D.). - The $1 / H$-variation of the divergence integral with respect to the fractional Brownian motion for $H>1 / 2$ and fractional Bessel processes. Stoch. Proc. Applications. 115, p. 289-289 (2005).

[18] Hu (Y.). - Integral transformations and anticipative calculus for fractional Brownian motions. Mem. Amer. Math. Soc. 175, (2005).

[19] Hu (Y.), Nualart (D.). - Some Processes Associated with Fractional Bessel Processes. J. Theoretical Probability 18, p. 377-397 (2005).

[20] Hu (Y.), ØKsendal (B.). - Fractional white noise calculus and applications to finance. Infin. Dimens. Anal. Quantum Probab. Relat. Top. 6, p. 1-32 (2003).

[21] Kolmogorov (A. N.). - Wienersche Spiralen und einige andere interessante Kurven im Hilbertschen Raum. C. R. (Doklady) Acad. URSS (N.S.) 26, p. 115-118 (1940). 


\section{David Nualart}

[22] Lyons (T.). - Differential equations driven by rough signals (I): An extension of an inequality of L. C. Young. Mathematical Research Letters 1, p. 451-464 (1994).

[23] Lyons (T.) and QIan (Z.). - System control and rough paths. Oxford University Press, Oxford (2002).

[24] Mandelbrot (B. B.), Van Ness (J. W.). - Fractional Brownian motions, fractional noises and applications. SIAM Review 10, p. 422-437 (1968).

[25] Millet (A.) and SAnZ-Solé (M.). - Large deviations for rough paths of the fractional Brownian motion. Preprint.

[26] Nourdin (Y.). - Thèse de doctorat. Université de Nancy I (2004).

[27] Nualart (D.), Pardoux (E.). - Stochastic calculus with anticipating integrands. Prob. Th. Rel. Fields 78, p. 535-581 (1988).

[28] Nualart (D.) and Rascanu (A.). - Differential equations driven by fractional Brownian motion. Collectanea Mathematica 53, 55-81 (2002).

[29] Nualart (D.), Rovira (C.), Tindel (S.). - Probabilistic models for vortex filaments based on fractional Brownian motion. Annals of Probability. 31, p. 18621899 (2003).

[30] Pipiras (V.), Taqqu (M. S.). - Integration questions related to fractional Brownian motion. Probab. Theory Rel. Fields 118, p. 121-291 (2000).

[31] Pipiras (V.), Taqqu (M. S.). - Are classes of deterministic integrands for fractional Brownian motion on a interval complete? Bernoulli 7, p. 873-897 (2001).

[32] Rogers (L. C. G.). - Arbitrage with fractional Brownian motion. Math. Finance 7, p. 95-105 (1997).

[33] Russo (F.), Vallois (P.). - Forward, backward and symmetric stochastic integration. Probab. Theory Rel. Fields 97, p. 403-421 (1993).

[34] Sкоrohod (A. V.). - On a generalization of a stochastic integral. Theory Probab. Appl. 20, p. 219-233 (1975).

[35] Sussmann (H. J.). - On the gap between deterministic and stochastic ordinary differential equations. Ann. Probability 6, p. 19-41 (1978).

[36] Young (L. C.). - An inequality of the Hölder type connected with Stieltjes integration. Acta Math. 67, p. 251-282 (1936).

[37] Z̈̈HLE (M.). - Integration with respect to fractal functions and stochastic calculus. I. Probab. Theory Related Fields 111, p. 333-374 (1998). 